\title{
The slang of young Russians and French: reinventing scientific linguistic collaboration in the age of coronavirus and containment
}

\author{
[Les argots des jeunes Russes et Français : réinventer la collaboration \\ linguistique scientifique a l'ere du coronavirus et du confinement]
}

\author{
Larisa G. Vikulova - Larisa V. Ukhova - Zhanna K. Gaponova - Lina V. \\ Razumova - Polina S. Ukhova
}

DOI: 10.18355/XL.2021.14.04.16

\begin{abstract}
From the beginning, remote work was considered in connection with social and environmental problems. The pandemic and coronavirus have left many teachers and researchers unable to experiment with online work. This new way of organizing work is at the heart of exciting research in various fields: remote collaborative science, imaging, training, and distance learning are an opportunity to demonstrate relevance and find new ways of working and interacting. This article is in the framework of a scientific project carried out over a year at a distance. He deals with the problem of learning the youth language (youth slang, the term is used in Russian linguistics) and aims to present the results of a comparative study of the structural and semantic features of shale of Russian and French youth. Language experiments are based on data from students of Yaroslavl State University and the University of Poitiers. The first part deals with the description of the procedure for collecting, verifying, and processing language data, as well as the methodology for collecting; it is based on psycholinguistic experiments, field studies, and sociological studies conducted in the student environment of the two countries. The second part of the article presents the results of the semantic and structural analysis of lexical units, morphological and semantic families representing argotisms (slanguismes, in Russian) of young people recorded during the survey. The derivational mechanisms used in French and Russian youth slang are studied and interpreted. This allowed the authors to discover language universals common to young Russian and French speakers, as well as to identify culturally relevant linguistic units capable of modelling and presenting the collective identity of Russian and French students, their language image of the world, and their language personality.
\end{abstract}

Key words: Youth slang, field research, derivative mechanisms, linguistic picture of the world, collective linguistic personality, universal and culturally marked language code, axiology, assessment

\section{Résumé}

Dès le début le travail à distance a été envisagé en lien avec des enjeux sociétaux et environnementaux. La pandémie et le Coronavirus ont eu pour effet de contredire un grand nombre d'enseignants et de chercheurs à expérimenter le télétravail. Cette nouvelle modalité d'organisation du travail est au cœur de recherches foisonnantes dans divers domaines : la science collaborative à distance, les visioconférences, la formation et l'enseignement à distance sont l'occasion de démontrer la pertinence et de trouver de nouvelles modalités de travail et d'interactions. Cet article se situe dans le cadre d'un projet scientifique mené depuis un an à distance. Il porte sur la problématique de l'exploration du langage des jeunes (le slang des jeunes, terme utilisé en linguistique russe) et se propose d'exposer les résultats d'une étude comparative des caractéristiques structurelles et sémantiques des argots des jeunes russes et français. L'expérimentation linguistique est basée sur les données des 
étudiants de l'université d'Etat de Yaroslavl' et de l'université de Poitiers. La première partie porte sur la description de la procédure de collecte, de vérification et de traitement des données linguistiques ainsi que sur la méthodologie de la collecte ; elle est basée sur des expériences psycholinguistiques, études de terrain et enquêtes sociologiques menées dans un environnement étudiant de deux pays. La seconde partie de l'article présente les résultats de l'analyse sémantique et structurelle des unités lexicales, des familles morphologiques et sémantiques représentant des argotismes (slanguismes, en russe) des jeunes relevés au cours de l'enquête. Sont étudiés et interprétés les mécanismes dérivationnels en usage dans l'argot des jeunes français et russes. Ceci a permis aux auteurs de découvrir des univers langagiers communs aux jeunes locuteurs russes et français, ainsi que de repérer des unités linguistiques culturellement pertinentes capables de modéliser et de présenter l'identité collective des étudiants russes et français, leur image langagière du monde et leur personnalité langagière.

Mots clé : argot des jeunes, études de terrain, mécanismes dérivationnels, image linguistique du monde (ILM), personnalité langagière collective (PLC), code langagier universel et marqué culturellement, axiologie, évaluation

\section{Introduction}

Dans le contexte de pandémie que nous traversons depuis un certain temps, des nouvelles modalités de travail se mettent en place. Il s'agit aussi d'une intensification de modalités déjà existantes : travail collaboratif, visioconférences, enseignement à distance. Deviendront-ils une norme demain ? Ces modalités seront-elles adaptées et utilisées d'une manière plus large au futur? Dans le présent article nous partageons les résultats d'un projet scientifique collaboratif avec l'université de Poitiers mené depuis deux ans dont un an à distance. Ceci nous permet de constater que le projet scientifique s'adapte au travail distancié. Les projets scientifiques des étudiants s'inscrivent bien dans la tradition des projets développée au sein de la pédagogie de projet.

Pour ce qui est de la linguistique il importe de signaler que nous assistons aujourd'hui à un tournant important de problématique. Nous pouvons parler en premier lieu de la linguistique de corpus, l'une des branches les plus prometteuses de la linguistique moderne. Les corpus deviennent ainsi un outil important d'analyse des pratiques langagières des locuteurs. Le second centre de gravité de la science moderne va vers les pôles fonctionnaliste et cognitif. Il s'agit d'une espèce de transfert d'intérêt scientifique qui va de la notion de "langue" à celle de la "parole" et de la notion du "système" à celle de 1’"usage" (Stepanov, 2008).

Ensuite, l'accent est mis de plus en plus souvent non sur les notions de « mot » et de « phrase ", mais sur celle du " discours » en tant qu'outil de communication réelle. Ainsi, les méthodes quantitatives et statistiques du langage acquièrent une grande importance. On manifeste aussi de l'intérêt pour les problèmes de variation de la langue en synchronie ; c'est un témoignage de l'attention portée aux facteurs psychosociaux du langage tandis que la notion de variabilité diachronique démontre un changement d'attitude à l'égard des notions de norme et de correction linguistique.

Notre étude s'inscrit dans certains de ces champs linguistiques d'investigation et se donne l'ambition de démontrer les possibilités de la méthode combinée de collecte des faits linguistiques développée lors de cette étude. Elle permet de donner une image plus structurée des phénomènes linguistiques, de leurs fonctions et caractéristiques, en particulier, celles de l'argot des jeunes, en tant que reflet de l'image linguistique du monde (ILM) de ses locuteurs.

XLinguae, Volume 14 Issue 4, October 2021, ISSN 1337-8384, eISSN 2453-711X 


\section{Literature Review}

L'argot est défini comme ensemble d'unités lexicales non codifiées, reconnues et utilisées par les groupes de référence comme signe d'isolement linguistique, ainsi que moyen d'expressivité lexicale par lequel les membres du groupe évaluent et expriment leur attitude envers la réalité environnante (Verain, 1981; François-Geiger, 1991; Valdman, 2000; Sokolija, 2002; Podhorná-Polická, Fiévet, 2006; Podhorná-Polická, 2007; Ukhova, 2012; Petteni, 2013; Warditz, 2013; Zheltukhina et al., 2017). Le système lexical argotique se caractérise par un renouvellement constant. Il se base sur des mécanismes traditionnels et innovants de changement, portant l'empreinte des innovations et de transgression des normes de la langue ; il se caractérise aussi par le " recyclage " du lexique commun et du lexique des argots traditionnels (Zhelvis, 1990; Kostyuk, 2002; Babai, 2012; Stepanov, 2014; Larina \& Ionova, 2015; Tameryan et al., 2020).

L'étude vise à identifier les caractéristiques structurelles et sémantiques de l'argot des jeunes étudiants (Volf, 2002; Ukhova, 2012), à systématiser et à décrire les mécanismes de sa formation, les domaines thématiques, à révéler son potentiel d'évaluation et à présenter les portraits linguistiques d'étudiants russes et français.

Dans le cadre de cet objectif un certain nombre de tâches lexicologiques a été défini :

- l'identification et l'établissement des fonctions de l'argot des étudiants,

- l'identification des champs thématiques, des mécanismes productifs de dérivation (specifiques et universels) d' argotismes utilisés dans la langues russe et française;

- la déscription du potentiel d'expressivité (Volf, 2002; Larina, 2015) des argotismes russes et français;

- la constitution de l'image de la personnalité langagière des étudiants russes et français à la base des corpus rassemblés.

Le but et les objectifs de l'étude ont défini la structure de l'article, qui comprend deux sections : méthodologique et linguistique. La première section décrit les étapes de la procédure de collecte, de vérification et de traitement du matériel discursif. La deuxième section est consacrée à la description des résultats principaux de l'analyse comparative des argotismes collectés.

\section{Methodology}

Le but a de décrire les principaux résultats de l'analyse comparative des argotismes recueillis.

\subsection{Procédure de Collecte et de Traitement des Données. Étapes, Objectifs et Hypothèses}

Dans la présente étude les auteurs ont utilisé un ensemble de méthodes de recherche scientifiques générales et spécifiques. Ainsi, à l'étape de la collecte de données, il s'agissait de mener une étude de terrain qui a permis de constituer les corpus russe et français, ainsi que de préciser les unités lexicales rassemblées à l'aide des méthodes statistico-lexicales. Parmi d'autres méthodes utilisées notons l'enquête, l'interview, l'observation en situation, l'enquête sur Internet (google forms), le classement des données.

Pour l'analyse et l'interprétation des données sont utilisées les méthodes d'observation et de description, l'analyse sémantique des champs lexicaux, les analyses sémasiologiques et onomasiologiques, l'analyse des entrées dictionnairiques, les méthodes mathématiques du traitement des données etc.

Les corpus russe et français ont été établis entre 2019 et 2020.

La procédure d'étude s'est déroulée en plusieurs étapes :

I. la collecte du matériel ;

II. la vérification des champs thématiques ;

III. la vérification des données ;

IV. l'interprétation des données. 
Les données linguistiques ont été recueillies à la suite d'une enquête menée auprès des étudiants des deux universités-partenaires : l'université d'Etat de Yaroslavl'(Russie) et de l'université de Poitiers (France). Dans la constitution du questionnaire nous nous sommes appuyés sur la méthode introspective élaborée à partir de l'étude de T.I. Retinskaya (2004) dans laquelle la linguiste identifie un certain nombre de champs thématiques de l'argot des étudiants français ; ils nous ont servi de point de départ dans notre propre analyse.

Certains changements ont été introduits dans la structuration des champs sémantiques, proposés par T.I. Retinskaya (2004). Ainsi, les champs lexicaux « nationalité » et « statut social » ont été réunis au sein du champ "être humain »; pareil pour les champs lexicaux " Police / Armes / Crime », "Fraude » et " Vol » réunis en un seul champ, celui de « Crime (Criminalité) ».

Le champ « laid » a été supprimé en raison de la présence du champ « beauté / laideur "dans la liste. Nous avons divisé ce dernier champ en deux. Nous partageons l'opinion de P. Monteil (1964), qui, dans son ouvrage Beau et laid en latin, parle de la possibilité d'analyser ensemble ces notions. Le linguiste explique sa position par le fait que «nous parlons de la même classe et que le lien entre les deux concepts est indéniable. On ne peut pas les considérer comme des antonymes au sens direct du mot ; il s'agit de deux qualités qui s'excluent mutuellement. Et cette propriété d'exclu mutuel les rend inséparables les unes des autres en termes d'expression de valeurs esthétiques »(Monteil, 1964: 11-12). Nous avons jugé trop abstrait le champ « mauvais ", qui renvoie à des référents hétérogènes et c'est pour cette raison que ce champ a été également retiré de notre liste des champs lexicaux étudiés.

Le questionnaire constitué comprenait une liste de champs réorganisés. Ils ont servi de base à une étude plus approfondie, visant la détermination à l'aide de la méthode introspective des domaines thématiques de l'argot des jeunes russe et français de la période étudiée. L'analyse effectuée a également contribué à la découverte des caractéristiques lexicales, sémantiques, axiologiques des argotismes identifiés.

L'emploi de la méthode introspective (qui remonte aux idées de L.V. Shcherba) est largement utilisé dans la linguistique russe. Elle repose sur l'idée d'acceptabilité des résultats d'études basées sur l'intuition du locuteur natif. L'introspection et la linguistique des corpus se complètent et peuvent être utilisées de pair dans les études linguistiques.

Nous notons également certaines limites de l'introspection et de la linguistique des corpus. Même si la méthode introspective peut bien servir pour identifier les unités linguistiques, toutefois elle ne fournit pas de données socio-contextuelles de leur utilisation. En revanche, la linguistique des corpus permet de prendre en compte l'aspect social. Cependant, les corpus peuvent souvent comprendre des textes qui ne couvrent pas toutes les zones d'activité linguistique des locuteurs. Ainsi, dans le cadre de notre étude, nous avons jugé approprié d'utiliser également les données des corpus disponibles sur Internet (corpus National de la langue russe (NCRL, 2020), ainsi que les corpus de la langue française (CFPP, 2000; ESLO, 2017; MPF, 2018).

100 étudiants de deux universités (50 de l'université d'Etat de Yaroslavl' et 50 de l'université de Poitier), ressortissants de différentes localités de la Russie et de la France âgés de 18-21 et faisant leurs études dans différentes filières universitaires ont participé à l'enquête.

Leur tâche consistait à choisir, au sein des champs thématiques proposés, les argotismes en usage quotidien. En outre, les répondants ont été invités à compléter la liste proposée des champs thématiques et/ou des unités argotiques. Si le répondant éprouvait des difficultés à proposer un nouveau champ lexical ou un nouveau lexème usité mais non classé dans les champs proposés il devait ignorer cette question.

Formulons les hypothèses de la présente étude :

XLinguae, Volume 14 Issue 4, October 2021, ISSN 1337-8384, eISSN 2453-711X 
- les résultats de l'enquête sont censés de permettre d'identifier à la fois les champs thématiques actifs ainsi que les champs moins usités ou absents du système argotique français ;

- les résultats de l'enquête devraient permettre de dresser une image langagière du monde de la jeunesse russe et française ;

- les argotismes découverts au cours de l'étude permettraient de créer une typologie des moyens dérivationnels utilisés régulièrement dans l'argot moderne des étudiants russes et français ; ils contribueraient également à systématiser et à structurer des moyens universaux et uniques réguliers (productifs) en russe et en français ;

- l'analyse du potentiel expressif des lexèmes collectés est susceptible de confirmer ou d'infirmer notre conviction que les argotismes utilisés dans le langage des jeunes possèdent une grande valeur expressive qui compte pour leur acceptation identitaire et coniventielle.

Les résultats de l'enquête ont été vérifiés à l'aide des dictionnaires modernes d'argot. Parmi les dictionnaires russes citons, en premier lieu, le Vocabulaire moderne $d u$ XXI ème siècle (Slovar' aktual'noj leksiki XXI veka) sous la direction de G.N. Sklyarevskaya (2006), le Dictionnaire de l'argot russe (Slovar' russkogo slenga) de B.S. Elistratov (2007), le Dictionnaire de l'argot des jeunes (Slovar' molodyozhnogo slenga) de S.I. Levikova (2003), DYS (2019), DMVJS (2014), le Dictionnaire d'argot français-russe et russe-français (Francuzsko-russkij i russko-francuzskij slovar' slenga) de A.E. Popkova (2013), les dictionnaires de l'argot français de J.P. Goudailler (2001) et de J.P. Colin (2006). Nous avons jugé correct d'introduire dans les corpus les lexèmes enregistrés comme argotiques au moins dans l'un de ces dictionnaires présentés supra.

Les résultats de l'étude ont permis de préciser les domaines thématiques utilisés dans la recherche, ainsi que de présenter le modèle de l'image langagière du monde des locuteurs argotisant français et russes. Afin d'analyser l'expressivité des unités argotiques relevées ont été utilisés les textes du corps National de la langue russe et les corpus de la langue de la jeunesse française (CFPP, 2000 ; ESLO, 2017; MPF, 2018).

L'expressivité des unités argotiques relevées a été évaluée et vérifiée grâce aux contextes d'utilisation repérés dans plusieurs sources : textes du corps National de la langue russe et les corpus de la langue de la jeunesse française (CFPP, 2000 ; ESLO, 2017; MPF, 2018), textes des réseaux sociaux Facebook.com et Vk.com, forums (http://www.jeuxvideo.com/forums/0-51-0-1-0-26-0-blabla-18-25-ans.htm, Entendu à l'université K.D. Ouchinsky (Podslushano v YAGPU im. K.D. Ushinskogo), https://vk.com/ntrek, "YAGPU K. D. Ouchinsky - GRAPHIE", "Inyaz, groupe de 317", "Prof typique (Tipichnyj prepod)", https://www.hip-hop.ru/forum, http://www.yaplakal.com/forum), ainsi que dans le cadre des recherches sur le terrain, menées en milieu étudiant (des enregistrements de la parole spontanée des étudiants ont été réalisés et décryptées).

Ainsi, les données linguistiques ont été recueillies en combinant des techniques d'introspection et de linguistique de corpus ; nous nous sommes appuyés aussi sur les données recueillies dans les dernières éditions des dictionnaires de vocabulaire non standard.

\section{Results and Discussion}

Les résultats de l'étude des caractéristiques structurelles et sémantiques de l'argot des étudiants russes et français.

1. La présente étude a permis de découvrir et de classer en fonction de leur fréquence les moyens de dérivation les plus usités dans l'argot des étudiants russes et français. L'analyse effectuée a permis aussi de formuler les conclusions suivantes :

Les mécanismes les plus usités dans la formation des argotismes russes sont : 
- la métaphorisation (bomba, tochit' (kushat'), visnut', razdavit' (vypit'), bronya, zharit'),

- la troncation (proga, chel, gash, sot, yaga, ava, klava, buk),

- l'emprunt (kovidivors, dumskrolling, lokdaun, tresh, spich, kul, bedboj, pati, fajt, kesh, epikfejl, shchit, shuzy, chika),

- l'idiomatisation (dat' hrapaka, vyjti v tirazh, prisest' na ushi, slovit' blednogo, prostuzhennyj na golovu, zakusit'sya yazykami),

- la troncattion + suffixe (korona (ot koronavirus), konina, uvazhushechka, ukurysh, viskarik, narik, tupen', klubnyak, dvizhuha),

- la suffixation (kovidlo (a), kovidka, kovidnyj, gryaznuha, vidos, babec, bratan, vkusnyashki),

- l'emprunt à l'argot des voleurs et malfaiteurs (zhmur, ment, musor, mochit', tusovka, her, chmo, loh),

- la métaphorisation + suffixe (sin'ka, torchok, vanil'ka, zhestkach, vpiska, nepruha, zakusit'sya).

$\mathrm{Au}$ sein de l'argot russe la problématique du coronavirus démontre une riche productivité des procédés suivants :

abréviation : kovid,

addition des racines : karantikuly, kovidiot,

métonymie : zumit'sya (utilisation de la plateforme Zoom pour la communication),

univerbation : distancionka, udalenka,

mimétisme : karantin, koronialy.

En français les plus usités sont :

- la métaphorisation (craquer sur qn, délirer, une prison (à propos de l'établissement d'enseignement), un navet (mauvaise Note), un flan (à propos de l'homme paresseux), planer, défoncé),

- l'emprunt (stone, swag, spliff, fight, des blacks, junkie (de l'anglais), la hess, la choune, la seum (de la langue arabe), des flics (de langue allemande), flouze, les groles, des condés,),

- l'idiomatisation (se mettre une murge, avoir une bonne descente, se faire du mauvais sang, avoir la tête de pou, avoir la pêche/la patate),

- la suffixation (connard, feignasse, pétasse, gratos ),

- le codage verlan (les keufs $<$ les flics, les meufs $<$ les femmes, la teuf $<$ la fête, un oinj<un joint),

- la troncation (les bourges $<$ bourgeois, la dèche $<$ déchéance, la zic $<$ music, dégeu $<$ dégénasse; corona, confifi - troncation et réduplication),

- la métonymie (le son (la musique), les bleus, les poulets, les nuggets (les policiers), une pervenche, la mouise, la panade (la pauvreté) etc.).

Tout comme en russe l'argot français des jeunes utilise par excellence:

- l'abréviation : kovid,

- l'addition des racines : coronabdos, covidiot, skypéro, whatsappéro, zoombar,

- le mimétisme : hypoconfiniaque (< hypocondriaque).

L'analyse effectuée a permis de découvrir dans les systèmes argotiques russe et français un certain nombre de traits similaires et divergents.

Notons tout d'abord que les mécanismes les plus usités dans les deux langues sont la métaphorisation, l'emprunt, l'idiomatisation, la troncation et la suffixation.

La métaphorisation touche principalement les verbes dans les deux langues. Par exemple, les verbes de la langue littéraire acquièrent dans la langue parlée des étudiants russes et français une signification différente («zhech'», "otzhigat'», «zaryvat'sya», «naryvat'sya», zumit'sya, " délirer », " planer », " arnaquer »).

XLinguae, Volume 14 Issue 4, October 2021, ISSN 1337-8384, eISSN 2453-711X 
L'emprunt est un autre mécanisme fréquent dans la formation des argotosmes. Cependant si la majorité des emprunts russes viennent uniquement de l'anglais, en français on constate un plus grand nombre de sources d'emprunts : l'arabe (une brêle $<$ beghel $=$ " mulet $»)$, le tsigane $($ chourrer $<$ tchorav $=$ "voler $»)$, les dialectes et les langues régionales (les groles < provençale grola $=$ " vieux soulier $»)$. On n'observe pas une telle diversité de sources en russe.

L'idiomatisation employée souvent dans les deux langues ne révèle pas de différence frappante. Précisons seulement que les expressions idiomatiques de l'argot des jeunes en russe et en français portent par excellence une connotation ironique «slovit' blednogo», "vyjti v tirazh», "avoir la tête de pou», «se faire du mauvais sang»).

En parlant de mécanismes en usage dans le processus de la formation des argotismes dans le langage des jeunes, il convient aussi d'envisager la métonymie ; elle fait partie de la liste des mécanismes principaux de formation des unités argotiques en français, alors qu'en russe, son utilisation est beaucoup plus modeste et apparaît souvent combinée à d'autres moyens, par exemple, à celle de la troncation et de la suffixation.

La métaphorisation combinée à la suffixation est plus fréquente dans l'argot des étudiants russes. On remarque que c'est le cas de la formation des argotismes des champs thématiques "Ivresse/dépendance ", " Loisirs ", "Querelle/bagarre ». En français cette combinaison des moyens n'est pas usitée si largement.

Les jeunes français préfèrent utiliser le codage verlan, jouant avec la forme des mots qui change par la permutation des syllabes. Les lexèmes d'un grand nombre de champs thématiques sont formés par verlanisation.

Il convient également de présenter brièvement le mécanisme d'addition des racines des mots. En français, il est présent sous sa forme pure (chinetoque, daron) ; en russe il apparait surtout combiné à l'emprunt («mordofajt», «kovidiot») et à la métaphorisation («govnoedy»).

Notons que la combinaison du plus grand nombre de moyens de formation est possible en français. Par exemple, quatre moyens sont utilisés pour former le mot «taspeche» : emprunt + codage verlan + troncattion + suffixe.

L'argot russe des jeunes puise beaucoup au langage cryptique des malfaiteurs, par exemple «pushka», «zhmur», «musor», «oper». Dans la langue française on découvre un certain nombre de lexèmes empruntés à l'argot théâtral, à celui des Zouaves et à celui de la typographie.

De cette façon on remarque cette année dans les deux langues l'activation des mécanismes auparavant peu usités de l'abréviation, de l'addition des bases, $d u$ mimétisme.

2. La seconde partie de notre étude a permis d'analyser les champs thématiques de l'argot des étudiants français et russes. Infra nous donnons les conclusions formulées lors de l'analyse des corpus.

1. L'argot des jeunes russes et français est déterminé par la présence de champs thématiques communs et forme leur image langagière du monde.

2. L'analyse effectuée a révélé des champs thématiques absents du système argotique des jeunes français (le champ «La technique ») et de l'argot des jeunes russes (le champ « Irritation, mécontentement »).

3. L'analyse du volume des champs thématiques (un volume total de 465 lexèmes) a permis d'identifier les champs thématiques les plus usuels de l'argot des jeunes russes.

L'étude effectuée a montré que le champ thématique "l'Homme et ses vices (ivresse et toxicomanie) » est un des plus usuel dans l'argot des jeunes russe.

Le champ thématique «Amitié / conversation amicale » est également assez usité. Un russe fait une distinction nette entre les lexèmes drug, priyatel', tovarishch, odnoklassnik, tandis que le concept d'ami n'a pas un tel degré de différenciation pour les jeunes français démontrant une compréhension différente et un autre degré d'intimité lié à ce mot dans les cultures russe et française. 
L'explication remonte aux facteurs sociaux qui doivent être pris en compte : en raison de la spécificité du système éducatif russe, les enfants étudient le plus souvent depuis dix ans dans la même école et parfois dans la même classe, se font des amis dès l'enfance, habitent souvent très proches les uns des autres, de l'école, passent beaucoup de temps ensemble, puis reçoivent souvent un enseignement supérieur dans la même université. L'amitié se renforce au fil des ans et fait partie intégrante de la vie des jeunes.

Le système éducatif français diffère sensiblement sur ce point. L'organisation plus distincte par niveaux : école primaire - collège - lycée - engendre déjà d'elle-même une mobilité plus accrue des élèves français. S'y ajoute une plus grande mobilité dans l'espace des familles françaises en général. L'enseignement supérieur oblige, lui aussi, les étudiants français à déménager plus souvent dans d'autres villes. Ainsi, les jeunes français n'ont pas la possibilité de se voir souvent avec des amis, de participer quotidiennement à leur vie. Cependant, ils font plus facilement de nouvelles connaissances, et s'en séparent aussi plus facilement. C'est probablement l'une des explications possibles pourquoi le champ lexical "Amitié / conversation amicale » reste inactif en français et se caractérise par une moindre étendue par rapport à la langue russe.

Un volume lexical considérable des champs thématiques « Apprentissage », « Loisirs / musique », " Beauté / laideur » témoignent du grand intérêt des étudiants russes pour ce sujet. Les problèmes du transport, de la maladie/mort, du logement éveillent peu d'intérêt parmi les étudiants russes. Ceci est démontré par le petit nombre de lexèmes faisant partie des champs thématiques correspondants (voir : Figure 1).

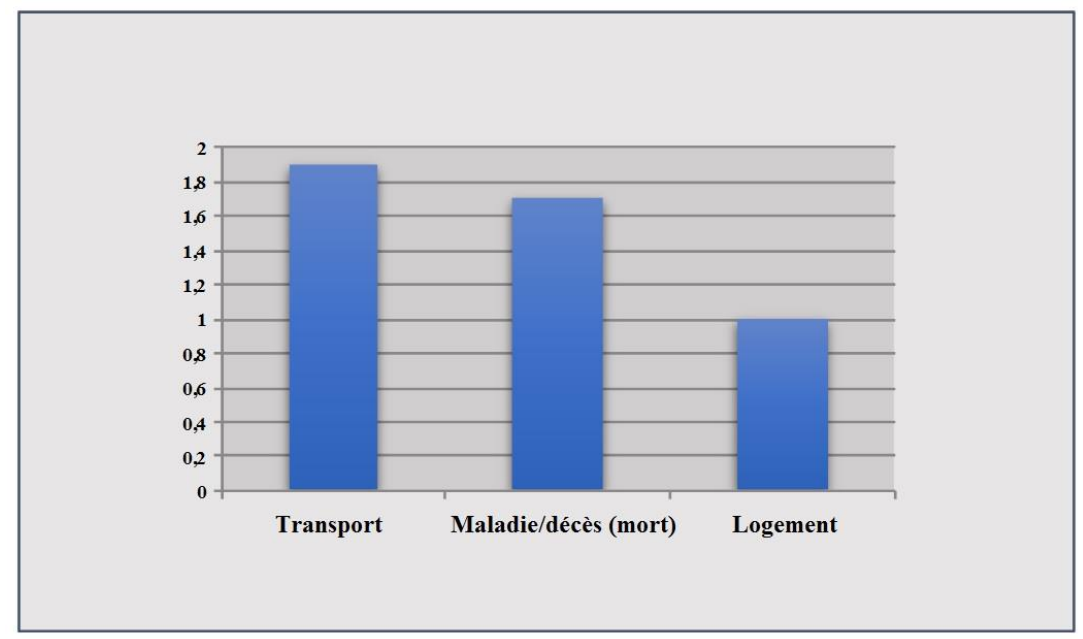

Figure 1. Les champs thématiques les moins actualisés du parler des jeunes étudiants russes

Ce phénomène est dû, parait-il, au fait que la majorité des jeunes étudiants en Russie vivent chez leurs parents, de sorte que les questions du loyer et des services médicaux ne sont pas à leur charge et, donc, restent à la périphérie de leurs intérêts. Il convient, toutefois, de noter que le champ thématique de la maladie par ces temps du coronavirus est devenu un peu plus volumineux en raison des unités lexicales signalées supra désignant cette nouvelle maladie et ses dérivés.

Dans le système de l'argot français des jeunes (377 lexèmes répertoriés), les domaines thématiques les plus populaires sont : "T oxicomanie / Ivresse 》, "Criminalité ", "

XLinguae, Volume 14 Issue 4, October 2021, ISSN 1337-8384, eISSN 2453-711X 
Stupidité / Folie 》, "Homme », " Beauté / laideur 》, "Apprentissage » (voir : Figure 2).

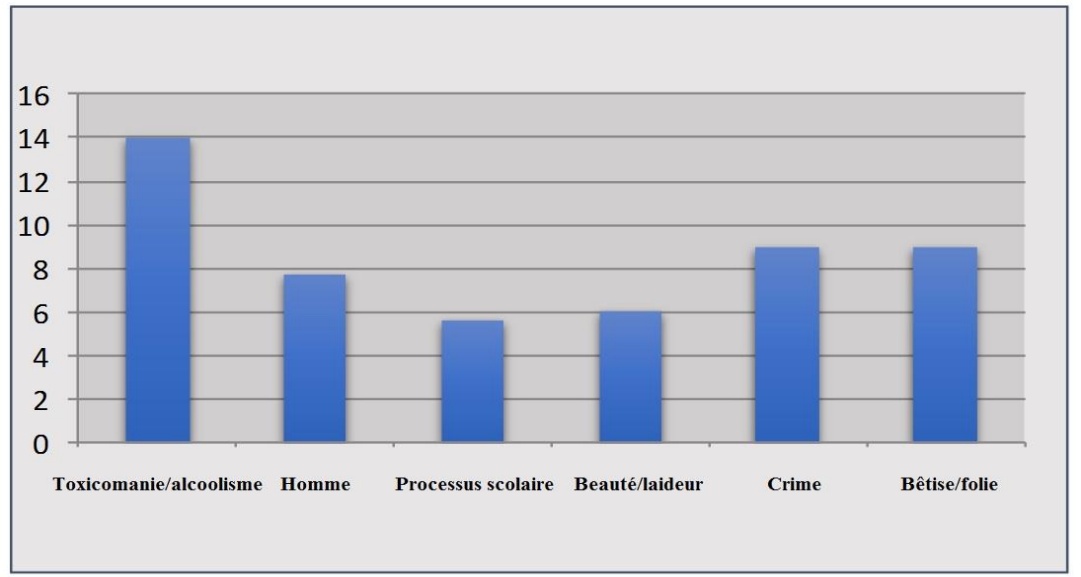

Figure 2. Les champs thématiques les plus actualisés du parler des jeunes français

Les moins populaires parmi les français sont les domaines du transport, du logement, de l'amitié.

Chez les jeunes français, comme chez les répondants russes, les champs «Drogue / Ivresse 》 et "Homme » étaient parmi les plus volumineux. Il est à noter toutefois que les étudiants russes proposaient davantage de lexèmes argotiques liés aux boissons alcoolisées et à leurs effets, tandis que les jeunes français désignaient plus des nominations des substances narcotiques, leur dose et leurs effets. L'analyse du champ "Homme ", où il a été suggéré d'indiquer l'âge, le statut social, le sexe et la nationalité, a donné des résultats intéressants. Contrairement aux étudiants russes qui ont indiqué des unités reflétant des relations familiales et amicales, les français ont donné de nombreux lexèmes se rapportant surtout à la richesse matérielle des personnes, et ayant des connotations péjoratives. Ceci nous mène à conclure que dans la mentalité des jeunes français il existe une attitude négative à l'égard de la démonstration ouverte de la richesse matérielle. C'est probablement l'une des explications de la présence dans le corpus de ce champs lexical.

Le champ " Beauté / laideur » figure également parmi les champs actifs de l'argot français des jeunes. Soulignons, cependant, que les jeunes français ont proposé plus de lexèmes à connotation positive (beau, physiquement fort etc.), tandis que parmi les répondants russes plus nombreux étaient les unités à connotation négative dénotant la laideur et les défauts physiques.

Pour ce qui est des défauts du caractère, du comportement et du niveau intellectuel, les étudiants français sont plus critiques et utilisent un plus grand nombre de lexèmes à connotation négative, péjorative, voire, grossière, selon l'étendue du champs thématique « Stupidité / Folie ».

Chez les répondants russes ce champ n'est pas tellement actif. Toutefois, il démontre aussi une étendue considérable incluant, par rapport aux champs respectifs français, des lexèmes à connotation ironique et moqueuse plutôt que grossière comme dans le cas de la majorité des lexèmes français du même champ.

Le champ "Criminalité" est également actif dans les deux systèmes argotiques. Les personnes interrogées ont été invitées à indiquer les lexèmes dénommant de différents types d'armes, de vol et pillage, ainsi qu'à nommer les policiers et leurs actions. Le 
champ constitué est volumineux en raison de ce que les deux langues manifestent une riche synonymie des unités lexicales désignant les représentants de la police de l'ordre publique. Nombreux sont les emprunts à l'ancien argot commun des malfaiteurs, dont la fonction principale était la fonction cryptique. Aujourd'hui, certains d'entre ces argots sont médiatisés dans les films policiers d'où ils pénètrent dans le langage des jeunes argotisants.

L'inactivité des champs thématiques «transport» et « logement » chez les jeunes Français s'explique aussi par des raisons extralinguistiques: le niveau de vie dans la plupart des cas permettant aux familles françaises de payer la location d'un logement ou d'une chambre d'étudiant ainsi que d'un véhicule à leur enfant, ces champs thématiques font rarement le sujet des discussions quotidiennes de jeunes français. Comme il vient d'être mentionné supra, aussi comme non actifs se présente dans le système argotique français le champ thématique « Amitié ».

Il est à noter que les répondants russes ont ajouté à la liste proposée initialement le champs thématique «Technologies nouvelles » ce qui nous parait tout à fait justifié vu l'impact que les technologies nouvelles d'information et de communication ont dans les sociétés modernes. Ceci s'explique aussi par le rôle croissant de l'argot informatique qui fait de plus en plus une partie intégrante de la langue des jeunes. Ce domaine, en outre, a également été complété par de nouvelles unités argotiques associées à l'utilisation de plates-formes en ligne pour l'apprentissage / l'éducation à distance largement utilisées cette dernière année en situation de pandémie et confinement. Les répondants français ont laissé ce champ sans attention, ajoutant à leur tour un nouveau champ thématique à la liste, celui de "Mécontentement / irritation ».

À la suite de l'analyse effectuée, il s'avère possible de brosser «le portrait » des jeunes locuteurs russes et français.

Ainsi, le jeune russe c'est une personne, faisant attention à son apparence, cherchant la perfection. Parmi les qualités qui peuvent être attribuées aux jeunes russes citons la spiritualité rattachant une grande importance aux valeurs éternelles telles que l'amitié, la famille et les relations humaines.

Le jeune français est défini par les qualités suivantes : la sûreté de soi-même, l'individualisme, la volonté de défendre ses droits et ceux des autres, il a un faible pour la critique, entre facilement en contact avec d'autres personnes, mais abandonne aussi facilement les contacts établis. Le confort dans tous les domaines occupe une place prépondérante dans sa vie.

3. La troisième section de notre analyse portait sur le dépoilement des lexèmes à valeur expressive (négative, neutre, positive, ambivalente) recueillis dans les corpus étudiés (le corpus national de la langue russe, les corpus français de l'argots des jeunes MPF, 2018; ESLO, 2017; CFPP, 2000). Une partie d'exemples a été trouvée sur les forums Internet et dans les réseaux sociaux ou proviennent d'études de terrain menées dans un environnement étudiant avec l'utilisation de la méthode d'observation participante. Leur analyse a permis de formuler les conclusions suivantes :

1) l'expressivité positive (représentée dans les corpus par 54 lexèmes russes et 39 français), est exprimée au sein des champs thématiques « Musique, loisirs », "Beauté ", « Amitié, conversation amicale », « Homme », " Amour, relations sexuelles ».

Dans le système des argotismes russes, nombreuses sont les unités lexicales formées par transfert métaphorique (Zheltukhina et al., 2020). L'utilisation de la métaphore permet au locuteur d'exprimer ses sentiments et ses émotions (cf.: «otzhigat'/zhech' na tuse», «ot etogo gashika normal'no shtorit») ainsi que son attitude envers le monde. La combinaison des moyens « emprunt + suffixe » est utilisée par les locuteurs russes pour exprimer une connotation ironique et plaisante en tant que moyen ludique de la parole (cf.: «respektuha», «frendit'», «bejbik»).

XLinguae, Volume 14 Issue 4, October 2021, ISSN 1337-8384, eISSN 2453-711X 
Les lexèmes tels que «kul», «nishtyak», «bomba» sont utilisés dans l'argot des jeunes russes comme signe d'approbation (par exemple, «bomba» peut se dire à propos d'un bon film, d'une belle chanson, des nouvelles captivantes ou à l'égard d'une belle fille).

L'étude des mécanismes dérivationnels en usage dans l'argot des jeunes français et russes a permis de formuler les conclusions suivantes.

Il existe un certain nombre de suffixes polyvalents employés largement dans l'argot des jeunes Russes en vue leur grand potentiel sémantique et combinatoire. Leur emploi permet aux locuteurs d'exprimer des rapports sémantiques variés allant de l'évaluation positive vers la désapprobation et la méprise. Ceux-ci incluent, parmi d'autres, les suffixes -иик (рэпчик), -ок (рэпачок), -ик (гарик). Les exemples indiquent que leur expressivité est due uniquement aux suffixes mentionnés. Sont

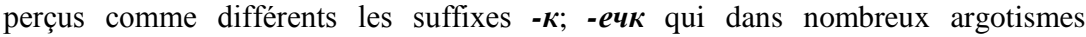
(«лафки», «уважушечка») jouent un rôle sémantique subsidiaire s'ajoutant comme complémentaire à l'expressivité contenue déjà dans la racine du mot qu'ils renforcent.

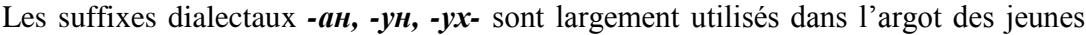
Russes. Ils appartiennent au langage familier et aux dialectes, et acquièrent une nuance conviviale, surtout dans les discours masculins des jeunes Russes (Vojvodina, 2007), par exemple : «братан», «дружбан», «корефан», «респектуха», "движуха». C'est aussi le cas, par exemple, du mot "респектуха" formé à partir de l'emprunt anglais "respect" qui en russe comme en anglais a une connotation positive. Ces suffixes servent également à composer des mots à connotation négative, ce qui confirme le fait qu'ils ne possèdent pas de sens propre mais sont utilisés par les locuteurs plutôt comme éléments asémantiques constructifs («грязуха» > du mot russe «грязь» (la boue) qui contient une connotation négative dans sa racine et qu'il conserve avec l'ajout du même suffixe $\boldsymbol{- y x}$ - ).

Dans l'argot français des jeunes, on retrouve également un grand nombre d'unités argotiques formées par emprunt et par transfert métaphorique. Dans les deux langues cela est dû au fait que le mot emprunté a une forme inhabituelle et attirante pour les jeunes parlant une autre langue. Le processus d'appropriation des emprunts passe par de nombreuses activités ludiques au cours desquelles les jeunes locuteurs jouent aux/avec les mots en les modifiant à leur gré. La métaphore leur permet d'exprimer toute une gamme d'émotions et d'attitudes envers le sujet de la parole (cf.: Kiff / kiffer (de l'arabe), branque (de branco), faire la chouille). Les combinaisons "emprunt + suffixation" et "emprunt + préfixation + suffixation", "emprunt + métaphore + préfixation + suffixation" sont également productives dans la formation des argotismes français à connotation positive. Dans les deux langues beaucoup d'argotismes sont empruntés à l'anglais qui est associé aux tendances innovantes des sociétés modernes. Les locuteurs de cultures et langues différentes utilisent largement les lexèmes anglais comme signe d'identité collective mais aussi comme signe d'appartenance langagière groupale (Zheltukhina et al., 2016). A l'utilisation du même langage et à la maîtrise du même lexique s'ajoute aussi l'utilisation d'un fond commun de moyens dérivationnels démontrant ce fait d'appropriation du nouveau lexique, y compris d'appropriation par transformation affixale. Par exemple, s'enjailler / enjaillement, dérivé du verbe anglais enjoy. Notons que les affixes mentionnés supra n'ajoutent pas, cependant, de valeur expressive supplémentaire au mot.

Un intérêt particulier relève l'utilisation du verlan dans le langage des jeunes français. Le verlan est un code de la langue qui remonte au XVI ${ }^{\text {ème }}$ siècle et qui à l'époque été utilisé afin de dissimuler le sujet de la conversation. Il a été ensuite oublié pendant plusieurs décennies, mais a été relancé par la suite. Les mécanismes de sa formation ont été bien décrit par P. Merle (2002) qui remarque que le verlan aujourd'hui n'est plus un code secret mais est utilisé par les jeunes en quête de forme originale du mot, par exemple, « gossebo »< « beau gosse », « faire la teuf » (« teuf »< « fête »). 
2) L'évaluation négative prévaut dans les deux corpus étudiés.

Dans le système argotique russe, ce groupe de lexèmes compte 184 unités, dont la plupart appartiennent au champ thématique "Education »(26 unités), ce qui s'explique par le fait que les étudiants consacrent beaucoup de temps à leurs études ; leurs activités et les problèmes discutés sont étroitement liés à l'éducation et se retrouvent constamment au cœur de leurs discours quotidiens sauvant « déguisés » en argotismes : «проспал», «химхлам»- sécher les cours ; «тюрьма»- université, «иило»- mauvaise note ou discipline).

Les champs thématiques «Stupidité » (26 unités), «Laideur 》 (25 unités), " Homme » (22 unités), "Lâcheté » (18 unités), "Criminalité » (18 unités) sont représentés par de nombreuses unités. Citons, par exemples, les lexèmes appartenant au champ " Stupidité " («затупок», "вообще тепльй», «вынос мозга), " Apparence " (стремный», «чушка», «грязнуха») et al. Le dernier champ témoigne du fait que la jeunesse russe est susceptible à l'apparence, s'efforce de suivre la mode, c'est pourquoi les vêtements sales et la mauvaise mine sont souvent proscrits dans le milieu des jeunes. Un grand nombre de mots liés au champ thématique "Homme" soulignent les défauts de l'éducation, le manque de culture, l'incapacité à communiquer : «azaльe», «быдло», «гопарь». Certains sont utilisés comme "indice » de la nationalité : «чурка», «узкодырый»; d'autres - de la lâcheté («ссыкло», «очковать», «каблук») ou du caractère criminel du comportement du locuteur («гонево», «лохануть», «мусарня», «замес», «закусон»).

Tout comme en russe, la plupart des unités argotiques françaises à connotation négative appartiennent aux champs thématiques mentionnés supra : "Stupidité" (23 unités : "barjo", "gourde", "chimique", "connard"), "Fatigue" (22 unités: « HS », « KO », « crevé »). Il est à noter que le champ thématique "Fatigue" jouit d'une plus grande popularité dans les discours quotidiens des jeunes français que de leurs homologues russes.

Les champs thématiques «top » des jeunes Français sont : «Education » (« une tôle/taule », " un navet », une " prison »), " Bagarre » ( " une mandale », " un gnon », « un coup de latte », " engueulade », « une baston »), " Echec » ( "Ras de bol! », " galérer », " avoir la poisse »), " Emotions, panique » ("flipper», " avoir les boules ", " se ronger les sangs ») et al.

Le champ thématique "Homme" propose lui aussi un grand inventaire d'unités lexicales argotiques (23 unités). Les lexèmes de ce champ sont utilisés pour prendre en dérision les personnes riches ("richards", "friqués", "joibourds"). Tout comme en russe, le champ contient des références à la nationalité ("chintoques", "bougnoules", "amerloques"). La signification négative et évaluative de la plupart des lexèmes de ce groupe est exprimée par les suffixes péjoratifs, cf. : -ard ( trouillard, richards); -oque ( vioque); -on- / -ron (laideron) ; -asse ( feignasse, pétasse) ; -os (craignos, tranquillos) ; - lo (dirlo). Les suffixes formatifs eur / -euse sont largement utilisés dans leurs discours pour former de nombreux mots à connotation négative : glandeur, branleur, emmerdeur, emmerdeuse.

Il apparait que le nombre des mots à connotation négative soit plus important dans les deux systèmes pour les deux raisons suivantes. Ainsi, V. I. Zhelvis note que dans la plupart des cultures nationales, les mots à connotation négative se retrouvent plus nombreux dans la parole que les mots positifs ; cette asymétrie s'explique dans la psychologie par le fait que les facteurs négatifs de la vie «dérangent » plus les humains et sont vécus par la personne d'une manière plus intense que les facteurs positifs qui sont généralement considérés comme naturels, normaux et donc moins émotionnels (Zhelvis 1990).

La deuxième raison est due, peut-être, au fait que de nombreuses unités de ce groupe sont connotées ironiquement. Grâce à ces argotismes les jeunes réalisent simultanément dans leur parole plusieurs effets : nominatif, expressif, ludique.

\footnotetext{
XLinguae, Volume 14 Issue 4, October 2021, ISSN 1337-8384, eISSN 2453-711X
} 
Ce groupe contient un vocabulaire évaluatif. C'est le cas des argotismes «иило», «отстой» / «отстойный», «лажа» / «лажовый», «борич», «сакс». Ces lexèmes se retrouvent dans plusieurs champs thématiques et du point de vue de leur potentiel combinatoire, ils apparaissent comme très usuels. C'est le cas, par exemple, de l'adjectif «oтстойный» qui peut être appliqué à une personne, à un animal, ou qui peut se dire à propos d'un film, d'une couleur etc. La même remarque peut être faite à propos du fonctionnement de l'argotisme «борш! !» qui peut être utiliser pour évaluer négativement l'apparence de quelq'un ou la qualité d'un objet.

3) Le groupe d'unités neutres comprend des emprunts au jargon des programmeurs, des toxicomanes, des étudiants ; ils sont utilisés comme termes et remplissent dans le discours une fonction nominative. Ce sont des unités appartenant aux champs thématiques "Technologie novelles ", " Matières, disciplines universitaires », " Argent », «Logement » («анал»- «вышка», «прога», «полка», «клава», « dоре », «tamien», «oinj »). L'argot français des jeunes utilise l'emprunt à l'ancien argot ("blé », " oseille ») ainsi qu'à l'anglais (" gun », " stone »). Le russe utilise lui aussi

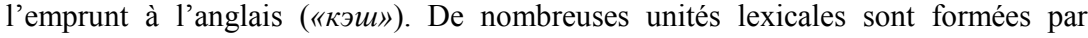
troncation («дек», «уни», «zic»).

4) L'analyse a permis de répertorier un certain nombre d'argotismes à signification ambivalente (69 unités en russe et 43 en français). Les argotismes russes de ce groupe ont été divisés en deux sous-groupes. Le premier comprend des unités dont la fonction est similaire à celle des interjections. Ces unités sont utilisées dans des phrases exclamatives («Жесть!», «Пипец!», «Tреu!») et expriment toute une gamme d'émotions (par exemple, «Жecmb!» qui peut être entendu, par exemple, comme réponse aux phrases " J'ai échoué à l'examen 》 ou "Hier j'ai dragué une fille 》 et al.).

Le second groupe d'argotismes comprend les unités polysémiques ayant une nuance grossière («бугай», «присесть на уши»), «трел», «мутки»); dans certains contextes leur emploie donne facilement la possibilité de passer d'une évaluation positive à une évaluation négative. Cela se rapporte, par exemple, au lexème «бyzaŭ (beuf)», cf.: "on ne s'est pas vu depuis longtemps. Eh bien, toi, le bœuf! Tu t'es engagé à bascule ?" ou «бугай (beuf)» est utilisé pour complimenter un jeune homme sportif aux muscles bien dessinés. Par contre, dans la proposition «Smotri tam kakoj bugaj na fejskontrole! (Regarde ce bœuf à l'entrée !)» le mot "бyzaŭ (beuf)» est utilisé dans le sens de « homme énorme à larges épaules présentant un danger avec qui il est mieux de ne pas communiquer » (Sklyarevskaya, 2006: 161).

La même observation peut être faite à l'égard du lexème «mutki» qui peut être utilisé dans le sens de "nouer une relation avec une fille » (Sklyarevskaya, 2006: 618) ou dans un sens opposé où "mutki» désigne une "affaire illégale " (le sens du mot remonte à l'adjectif mutnyj «impur, illégal») (Sklyarevskaya, 2006: 618).

Les unités lexicales françaises à valeur évaluative ambivalente ont été également divisées en deux groupes. Le premier comprenait des unités qui, selon le contexte, sont capables d'exprimer à la fois des significations négatives et positives, ainsi que des nuances de sens supplémentaires : admiration, colère, etc. Par exemple, le Tarot! Cinglé!», Enfoiré!» signifie "malade / fou / fou"). Ces adjectifs peuvent être utilisés dans certains contextes comme des insultes ("Mais il est complétement taré, celui-ci ! $\mathrm{Ne}$ vous laissez-pas faire, les filles, on vous soutient. Aucun respect, aucune délicatesse !". Dans d'autres, ils sont capables d'exprimer l'admiration ("Mais il est taré mdrrrr, il m'a tué, oh la blague !" - "Ahah, comme il est fou ! Il vient de me tuer avec sa blague !"). Cependant, il est impossible de parler d'un changement axiologique complet (Henriette, 2001; Arnaud, 2012). C'est le cas de l'argotisme "taré" qui même dans les contextes positifs conserve néanmoins des connotations négatives (Podhorná-Polická, 2007).

Le même groupe comprend deux autres unités lexicales qui changent de signe selon le contexte. Ainsi, le lexème accro est formé en tronquant l'adjectif accroché, qui 
désigne au sens figuré une personne qui est profondément amoureuse de quelqu'un ou de quelque chose ou qui est dépendante (y compris, de la drogue). Ainsi, quand il s'agit de tout type de dépendance (alcool, drogue, dépendance d'Internet), le lexème acquiert une connotation négative, souvent péjorative (" Depuis qu'il a douze ans il fume du shit, c'est un truc de ouf, il est accro ! »). Dans des contextes où il s'agit de sentiments d'une personne le mot peut obtenir des connotations à la fois positives et négatives (cf.: "Je suis vraiment accro de mon mec ! C'est l'homme de ma vie !" //" Je suis accro de lui, complétement dépendante, ça me fait peur, j'en viens à la dépression !") (Podhorná-Polická, 2007: 443).

Le deuxième groupe comprend les argotismes avec une expressivité ambivalente manifestée, comme en russe, dans le champs thématique «Toxicomanie, alcool». Les répondants ont suggéré un certain nombre de synonymes pour le verbe «boire », dont la connotation dépend principalement du sujet de l'évaluation, de ses normes comportementales et morales et de ses attitudes, ainsi que de la situation d'évaluation dans son ensemble. Ainsi, l'expression « se mettre une race / une murge / se bourrer la gueule » / « se saouler» ou, comme disent les jeunes russes «нажраться в слюни»" s'enivrer de salive») peut avoir une connotation ambivalente. Dans certains contextes, tous ces verbes ont le sens « boire beaucoup ", dans d'autres les mêmes verbes seront perçus comme vulgaires, grossiers contenant une touche de reproche. Le sujet, l'objet de l'évaluation et la situation de l'évaluation dans son ensemble jouent un rôle capital dans le processus de l'évaluation.

Ainsi, les nouveaux défis jetés par la pandémie du coronavirus, les changements, similaires, qui se sont opérés à l'échelle mondiale ont révélé des tendances similaires, elles aussi, dans les systèmes argotiques des étudiants russes et français : utilisation d'internationalismes, en règle générale, tels que Zoom- (notons que dans les langues anglophones, l'utilisation d'un élément peut être accompagnée d'une graphohybridisation), COVID-, Corona. En outre, l'argot des étudiants russes et français exploite largement leurs propres systèmes d'affixes - d'origine slave ou française.

\section{Conclusion}

La méthode combinée de collecte de données linguistiques présentée dans ce travail permet d'envisager plusieurs types d'énoncés - langage oral spontané, textes recueillis de différentes sources, corpus nationaux et thématiques, forums et al. Elle fait appel à l'intuition des locuteurs et permet de soumettre à l'analyse des sources et approches variées ce qui a sans doute un impact positif sur l'étude.

Les normes de la culture, les normes de la langue, toute norme sociale coordonne le comportement de ces membres assurant leurs intégrités. Les moyens expressifs de la langue sont eux aussi l'un des « instruments de régulation » des contacts entre les membres de la société. L'argot en tant que système expressif du langage remplit une fonction intégrative désignant, dénotant et encourageant les « siens », les « amis » en les séparant de ceux qui sont considérés comme " étrangers ». L'argot est également une image réfléchie de la culture nationale, car chacune d'entre elles contribue différemment à la manifestation de la même émotion humaine. En d'autres termes, différentes ethnies peuvent souvent utiliser le même code pour exprimer des émotions différentes, ce qui constitue l'objet de l'énantiosémie interethnique. C'est cette discipline qui présente un intérêt particulier ; certains de ses éléments ont été employés dans cette étude comparative basée sur l'idée que tout système argotique en tant qu'instrument d'évaluation est à la fois l'expression des valeurs de ses usagers. Le vocabulaire expressif recueilli lors de la présente étude révèle des différences culturelles dans les systèmes argotiques de la jeunesse française et russe.

L'argot des jeunes reflète l'image langagière que les jeunes se font sur leur monde, c'est une image réductrice, certes, mais contenant des moyens intéressant de

XLinguae, Volume 14 Issue 4, October 2021, ISSN 1337-8384, eISSN 2453-711X 
construction lexicales, des sujets favoris (chouchou) et ses moyens expressifs qui tous ensemble leurs donnent la possibilité de s'approprier la langue, de la sentir " maîtrisée » et « domptée ».

\section{Acknowledgments}

The publication has been prepared with the support of the Yaroslavl State Pedagogical University named after K.D. Ushinsky.

\section{Bibliographic references}

ARNAUD, C. 2012. Axiologie 4.0: proposition pour une nouvelle axiologie. Lyon: PUF. Available online: https://www.axiologie.org. ISBN: 978-2-7466-4295-9.

BABAI, L.V. 2012. Intercultural communication in the context of globalization: $\mathrm{PhD}$ paper in Philology. Kharkov: KhSU.

CFPP. 2000. Corpus de Français Parlé Parisien des années. Available online: http://cfpp2000.univ-paris3.fr/Corpus.html

BENABID, F. 2021. Lexicovid-19, une floraison de nouveautés linguistiques. In: Synergies Algérie, Vol. 29, 2021, pp. 161-175. ISSN 1958-5160 ISSN

COLIN J.P. 2006. Argot \& français populaire. Paris: Larousse. ISBN 2035323118.

DMVJS. 2014. Dictionary of Modern Vocabulary, Jargon and Slang. Available online: https://argo.academic.ru/

DYS. 2019. Dictionary of youth slang. 2019. Available online: http://teenslang.su

ELISTRATOV, B.S. 2007. Interpretive dictionary of Russian slang. Moscow: ASTPress Book. ISBN 5-462-00441-9.

ESLO. 2017. Corpus des ESLO (Enquête SocioLinguistique à Orléans). Available online: http://eslo.huma-num.fr/index.php/pagecorpus/pageacces corpus

FRANÇOIS-GEIGER, D. 1991. Panorama des argots contemporains. In: Langue française, 90, pp. 5-9. ISSN 0023-8368. e-ISSN 1957-7982.

GOUDAILLER J.P. 2001. Comment tu tchatches! Dictionnaire du français contemporain des cités. Paris: Maison neuve et la rose. ISBN: 2-7068-1476-4.

HENRIETTE, W. 2001. Axiologie et sémantique chez André Martinet. In: La linguistique, vol. 37, pp. 59-68. ISBN: 9782130520474.

KAREK,M. 2019. L'acquisition de la compétence lexicale à l'école moyenne en Algérie : problèmes liés aux consignes. In: Synergies Algérie, Vol. 27, pp. 1531. ISSN 1958-5160

KOSTYUK, O.V. 2002. Intercultural communication in the process of globalization of the modern world: PhD Thesis. Stavropol: SSU.

LARINA, T.V. - IONOVA S.V. 2015. Linguistics of emotions: from theory to practice. In: Bulletin of the Peoples' Friendship University of Russia. Series: Linguistics. Moscow: RUDN. ISSN 2312-9182.

LARINA, T.V. 2015. Pragmatics of emotions in an intercultural context. In: Bulletin of the Peoples' Friendship University of Russia. Series: Linguistics. Moscow: RUDN. ISSN 2312-9182.

LEVIKOVA, S.I. 2003. Large dictionary of youth slang. Moscow: Grand: Phairpress. ISBN 5-8183-0673-9.

MERLE P. 2002. Précis de français précieux au XXIe siècle. Paris: Renaissance du livre. ISBN 9782804606435.

MONTEIL, P. 1964. Beau et laid en latin, étude de vocabulaire. Paris: Klincksieck. ISBN-10: 2252005017.

MPF. 2018. Corpus Multicultural Paris French. Available online: https://www.ortolang.fr/market/corpora/mpf

NCRL. 2000. National Corps of the Russian Language. Available online: http://v.ruspora.ru. 
PETTENI, O. 2013. L'argot dans les discours hugolien et célinien. Une lecture symptômale des apories du concept de fraternité. In : Dissensus [En ligne], Varia, n. 5. e-ISSN 2031-4981. Available online: https://popups.uliege.be/20314981/index.php?id=1363.

PODHORNA-POLICKA, A. - FIEVET, A.-C. 2006. Les médias, l'argot et l'imaginaire argotique - une comparaison franco-tcheque (Mass-media, slang and slang's imagination - comparision of French and Czech youth slang). In : Revue d'Etudes Francaises, vol. 11, n. 1, pp. 27-52. ISSN 1416-6399.

PODHORNA-POLICKA, A. 2007. Peut-on parler d'un argot des jeunes? Analyse lexicale des universaux argotiques du parler de jeunes en lycées professionnels en France (Paris, Yzeure) et en République tchèque (Brno). Paris: Université Paris V René Descartes.

POPKOVA, A.E. 2013. French-Russian and Russian-French dictionary of slang. Moscow: Living language. ISBN 978-5-8033-0860-7.

RETINSKAYA, T.I. 2004. Sources and mechanisms for the formation of the French student argo: PhD Thesis. Moscow: MSU.

SERIDI, H. - BOUDECHICHE, N. 2020. Conscientisation du phénomène collocationnel : de la prise de conscience au savoir-faire langagier. In: Synergies Algérie, Vol. 28, pp.175-192. ISSN 1958-5160

SKLYAREVSKAYA, G.N. 2006. Explanatory dictionary of the Russian language of the beginning of the XXI century. Actual vocabulary. Moscow: Exmo. ISBN 978-5699-15913-0.

SOKOLIJA, A. 2002. Étude contrastive des argots de sarajevo et de paris. Aspects méthodologiques. In: La linguistique, vol. 38, n. 1, pp. 99-112. ISSN 2101-0234.

STEPANOV, V.N. 2008. Pragmatics of spontaneous television speech. Yaroslavl: MUBiNT. ISBN 5-93002-177-5.

STEPANOV, V.N. 2014. Emotionogenic speech and its genres in advertising. In: Psychological influence in interpersonal and mass communication. Moscow: Publishing House "Institute of Psychology of the Russian Academy of Sciences. ISBN 978-5-9270-0286-3.

TAMERYAN, T.Y. - ZHELTUKHINA, M.R. - ANIKEJEVA, I.G. ARKHIPENKO, N.A. - SOBOLEVA, E.I. - SKUYBEDINA, O.N. 2020. Language explication of the conceptualized meanings in ethno-cultural and socio-political aspects of discourse. In: Opcion, vol. 26, pp. 456-475. ISSN 1012-1587.

UKHOVA, L.V. 2012. Stereotype and creativity of the advertising text: to the question of the effectiveness of communication. In: Stereotyping and creativity in the text. Inter-university collection of scientific works. Perm: Perm State National Research University. ISBN 978-5-7944-2232-0.

VALDMAN, A. 2000. La Langue des faubourgs et des banlieues: de l'argot au français populaire. In: The French Review, vol. 73, n. 6, pp. 1179-1192. ISSN 0016-111X.

VERAIN, J. 1981. Les malades $\mathrm{du}$ taquet Argot des médias ou sociolecte des motards ? In: Journal of Lexicology. Cahiers de lexicologie vol. 2, n. 39, pp. 105-127. ISSN 2262-0346. Available online: https://doi.org/10.15122/isbn.978-2-8124-4292-6.p.0107

VOJVODINA, G.A. 2007. Unique affixes in modern Russian: Educational and methodological manual. Yelets: YSU named after I.A. Bunin.

VOLF, E.M. 2002. Functional evaluation semantics. Moscow: Individual URSS. ISBN 978-5-397-00803-7.

WARDITZ, V. 2013. Zur linguistischen Rezeption des französischen Romans in Russland: Das Phänomen des Argots in Hugos Les Misérables und in Krestovskijs Peterburgskie truščoby. In: Zeitschrift für Slawistik, vol. 58, n. 4, pp. 391-416. ISSN: 0044-3506. e-ISSN 2196-7016. Available online: https://doi.org/10.1524/slaw.2013.58.4.391

XLinguae, Volume 14 Issue 4, October 2021, ISSN 1337-8384, eISSN 2453-711X 
ZHELTUKHINA, M.R. - MAGOMADOVA, T.D. - CHERVYAKOVA, L.D. KOROVINA, S.G. - KRIVOSHLYKOVA, L.V. - NESOVA, N.M. 2020. Military metaphor as means of modern media knowledge communication: functional, lexical and grammatical aspects. In: Astra Salvensis, Supplement, vol. 1, pp. 515-547. ISSN 2457-9807. ISSN-L 2457-9807

ZHELTUKHINA, M.R. - VIKULOVA, L.G. - MIKHAYLOVA, S.V. BORBOTKO, L.A. - MASALIMOVA, A.R. 2017. Communicative Theatre Space in the Linguistic and Pragmatic Paradigm. In: XLinguae Journal, vol. 10, pp. 85-100. ISSN 1337-8384.

ZHELTUKHINA, M.R. - VIKULOVA, L.G. - SEREBRENNIKOVA, E.F. GERASIMOVA, S.A. - BORBOTKO, L.A. 2016. Identity as an Element of Human and Language Universes: Axiological Aspect. In: International Journal of Environmental and Science Education, vol. 11, n. 17, pp. 10413-10422. eISSN 13063065 .

ZHELVIS, V.I. 1990. Emotive aspect of speech. Psycholinguistic interpretation of speech impact. Yaroslavl: Publishing House of YAGPI named after K.D. Ushinsky.

ZHILAVSKAYA, I.V. 2016. The quality of journalism as a media education program. In: Vestnik of Volzhsky University after V.N. Tatischev, n. 3, pp. 120-125. ISSN 2076-7919.

Words: 8949

Characters: 59728 (33,18 standard pages)

Prof. Larisa Georgievna Vikulova, Dr.Sc.

Associate Prof. Lina Vasilevna Razumova, DrSc.

Foreign Languages Institute

Moscow City University

5 b Maly Kazenny per.

105064 Moscow

Russia

vikulovalg@mail.ru

lina.razumova@mail.ru

Associate Prof. Larisa Vladimirovna Ukhova, Dr.Sc.

Associate Prof. Zhanna Konstantinovna Gaponova, PhD

Faculty of Russian Philology and Culture

Yaroslavl State Pedagogical University named after K.D. Ushinsky

108/1 Respublikanskaya Str.

150000 Yaroslavl

Russia

larissauchowa@mail.ru

jangap1@mail.ru

Lector Polina Sergeevna Ukhova, $\mathrm{PhD}$

Faculty of Foreign Languages and Civilizations

Bordeaux-Montaigne University

19, esplanade des Antilles

33607, Pessac

France

polina.ukhova90@gmail.com 